\title{
LAS INTENCIONES DE COMPORTAMIENTO DEL ESTUDIANTADO UNIVERSITARIO HACIA LA GESTIÓN DE LA SOSTENIBILIDAD
}

\section{THE BEHAVIORAL INTENTIONS OF THE STUDENTS TOWARDS THE MANAGEMENT OF SUSTAINABILITY}

\begin{abstract}
ב" Juan Isidro Escámez Marsilla $a^{a^{*}}$ y Juan Escámez Sánchez
\end{abstract} Fechas de recepción y aceptación: 15 de abril de 2020 y 28 de abril de 2020

DOI: 10.46583/edetania_2020.57.608

Resumen: El objetivo es explorar las intenciones de comportamiento del estudiantado del Grado de Administración y Dirección de Empresas de dos universidades valencianas como agentes de sostenibilidad; el interés de tal objetivo es debido a que las intenciones predicen las conductas. Para ello elaboramos un cuestionario-escala, según la Teoría del Comportamiento Planificado de Ajzen, que aplicamos a una muestra del estudiantado e interpretamos sus respuestas según la metodología hermenéutica crítica. Las conclusiones más significativas: hay más alumnado con intención positiva que negativa hacia la sostenibilidad; es mejor en las mujeres que en los varones; el porcentaje de alumnado con intención negativa e indiferente es elevado y exige programas específicos de formación.

Palabras clave: estudiantado universitario, agente de sostenibilidad, teoría del comportamiento planificado, hermenéutica crítica, intenciones de comportamiento.

Abstract: This paper aims to explore the behavioral intentions of the students at the Degree in Business Administration and Management from two Valencian universities. Students were understood as agents of sustainability where the intentions predict behavior. A questionnaire-

a Oficina de Relaciones Internacionales. Universidad Católica de Valencia, San Vicente Mártir.

Correspondencia: Universidad Católica de Valencia San Vicente Mártir. Oficina de Relaciones Internacionales. Calle Guillem de Castro, 94. 46001 Valencia. España.

E-mail: ji.escamez@ucv.es

${ }^{\mathrm{b}}$ Doctor en Filosofía. Catedrático de Universidad. 
scale was developed, according to Ajzen's Planned Behavior Theory. A sample of students provided answers according to the critical hermeneutical methodology that were interpreted afterwards. Conclusions are described as follows: Positive intentions were higher than negative ones towards sustainability; women referred better intentions; the percentage of students with negative and indifferent intention is high and requires specific training programs.

Key words: university students, agent of sustainability, theory of planned behavior, critical hermeneutics, behavioral intentions.

\section{INTRODUCCIÓN: ANTECEDENTES Y FUNDAMENTOS DE LA INVESTIGACIÓN}

Para el asunto que interesa de modo especial en este artículo, el diagnóstico de las intenciones de los universitarios del grado de Administración y Dirección de Empresa para la gestión de la sostenibilidad, se han presentado dos iniciativas internacionales: el Pacto Mundial para el Desarrollo de un Mundo Sostenible (ONU, 2004), que consiguió durante el año 2018 la adhesión de más de 13.000 entidades firmantes de 145 países, está basado en declaraciones y convenciones internacionales ${ }^{1}$ aplicadas en cuatro áreas: derechos humanos, medio ambiente, estándares laborales y anticorrupción. La segunda iniciativa, Principios para una Educación Responsable en Gestión (ONU, 2007), persigue organizar las relaciones entre la Oficina que gestiona el Pacto Mundial y las Facultades de Economía, Administración y Dirección de Empresas y/o Escuelas de Negocios.

En cuanto a la necesidad de que el aprendizaje educativo responda a los retos actuales más urgentes a los que se enfrenta el planeta y la comunidad internacional, el informe liderado por I. Bokova, Replantear la educación. ¿Hacia un bien común mundial? (Unesco, 2015), defiende que la finalidad de la educación del siglo xxI tiene que centrarse en preparar a los individuos y a las sociedades para responder a la complejidad y a las contradicciones que hacen insostenible el mundo en el que vivimos. La formación como agentes de sostenibilidad tiene que ser uno de los objetivos básicos de la educación actual (ONU, 2015a; Unesco, 2017). La importancia de la formación de los

${ }^{1}$ La Declaración Universal de los Derechos Humanos, la Declaración de la Organización Internacional del Trabajo sobre los Principios Fundamentales y Derechos en el Trabajo, la Declaración de Río sobre el Medio Ambiente y Desarrollo y la Convención de Naciones Unidas contra la Corrupción. 
ciudadanos como agentes de desarrollo sostenible también se ha evidenciado en la Convención Marco sobre el Cambio Climático del 12 de diciembre de 2015 en París (ONU, 2015b).

El enfoque con el que las universidades han respondido a las iniciativas de la ONU, para formar a los estudiantes como agentes de sostenibilidad, ha sido fundamentalmente la introducción en sus planificaciones curriculares de cuestiones o disciplinas sobre ética de los negocios o responsabilidad social corporativa. La revisión mundial que han realizado los profesores SetoPamiés y Papaoikonomou (2016) ofrece una perspectiva interesante sobre el estado actual del aprendizaje-enseñanza universitario sobre sostenibilidad.

También ofrecen interés los informes internacionales auspiciados por la ONU, liderados por Haski-Leventhal (2012, 2013, 2014, 2015, 2016), sobre los Principios para una Educación Responsable en Gestión, basados en encuestas a los estudiantes de las escuelas de negocios y facultades de Administración y Dirección de Empresas (ADE) y/o Economía, que manifiestan la importancia que el estudiantado concede al desarrollo moral para un comportamiento sostenible. Nos ha sido de especial interés el informe del año 2015 (Haski-Leventhal, Pournader y Mckinnon) en el que se llama la atención sobre la variable género, puesto que muestra diferencias significativas entre mujeres y varones; las estudiantes manifiestan intenciones de comportamiento sostenible más positivas que sus compañeros varones. Queremos verificar si también es así entre el estudiantado de las dos universidades valencianas (España) objeto de la presente investigación.

Son muchos los investigadores que defienden que la principal misión del Pacto Mundial es conseguir valores compartidos y normas morales para orientar la gestión de la economía mundial (Williams, 2014). La promoción de la dimensión ética en las empresas y la Responsabilidad Social Corporativa viene incrementándose en los currículos universitarios, tanto por la Unión Europea (Lozano, 2012) como por las políticas educativas de otras regiones del mundo, como se manifiesta en los planes estratégicos de más de cuarenta instituciones canadienses (Henderson, Bieler y Mckenzie, 2017).

Quizás, lo más interesante de esas investigaciones sean las señales que nos orientan sobre el camino que debamos seguir en el futuro: la primera señal es que la sostenibilidad se analiza desde la perspectiva del desarrollo sostenible; concepto flexible según los contextos en el que se utiliza, sea la educación, las 
tecnologías, las ciencias experimentales, la economía, la protección del medio ambiente, las políticas públicas o los derechos sociales, y los escenarios en los que se aplica sea global, regional, institucional o personal (SederevičiūtèPačiauskienė y otros, 2017). La segunda señal es la argumentación de que el desarrollo sostenible va unido a la calidad de vida de los individuos y de las naciones (Nussbaum y Sen, 1996). La tercera señal es que el problema central de la Educación Superior consiste en que los estudiantes aprendan las capacidades y/o competencias como agentes de sostenibilidad y adquieran un verdadero compromiso para su práctica (UNESCO, 2017).

El diagnóstico de tal compromiso es lo que explora esta investigación. Para la elaboración del instrumento que nos permita un diagnóstico fiable hemos seleccionado una de las teorías más aceptadas por los científicos sociales de nuestro tiempo: la teoría del Comportamiento Planificado de Ajzen, que tiene un consolidado prestigio, y está avalada con una prolongada práctica de la investigación empírica (Steinmetz, Davidov y Schmidt, 2011; Steinmetz, Knappstein, Ajzen, Schmidt y Kabst, 2016).

Presentamos a continuación algunas consideraciones que, a nuestro juicio, son de especial importancia para argumentar la adopción de esa teoría y no de otra. Las consideraciones están referidas a las siguientes cuestiones: 1) la concepción de persona y del comportamiento social humano; 2) los elementos que conforman las intenciones de comportamiento; 3) los criterios que hay que tener en cuenta para que las intenciones de comportamientos sean predictoras de conductas; y 4) cómo se pueden probar empíricamente las afirmaciones que se hacen y los programas de formación de los estudiantes como agentes de sostenibilidad.

El fundamento clave de la teoría es la concepción de la persona como esencialmente racional, que usa la información puesta a su disposición, desde la interacción con sus contextos, para hacer juicios, evaluaciones y tomar decisiones. Sostiene que la conducta social humana no está determinada por motivos inconscientes, deseos irresistibles, creencias caprichosas o irracionales, sino que, por el contrario, el individuo considera las consecuencias positivas o negativas para él de su acción antes de decidirse en su desempeño. De ahí el nombre de teoría del Comportamiento Planificado, puesto que el sujeto controla su propia conducta sirviéndose de la información como base de su decisión. Con todo rigor puede decirse que la teoría adopta una perspectiva 
cognitiva para comprender las intenciones y predecir las conductas (Escámez, 2019). Gráficamente la teoría se representa así:

FIGURA 1

Elementos que conforman la teoría del Comportamiento Planificado

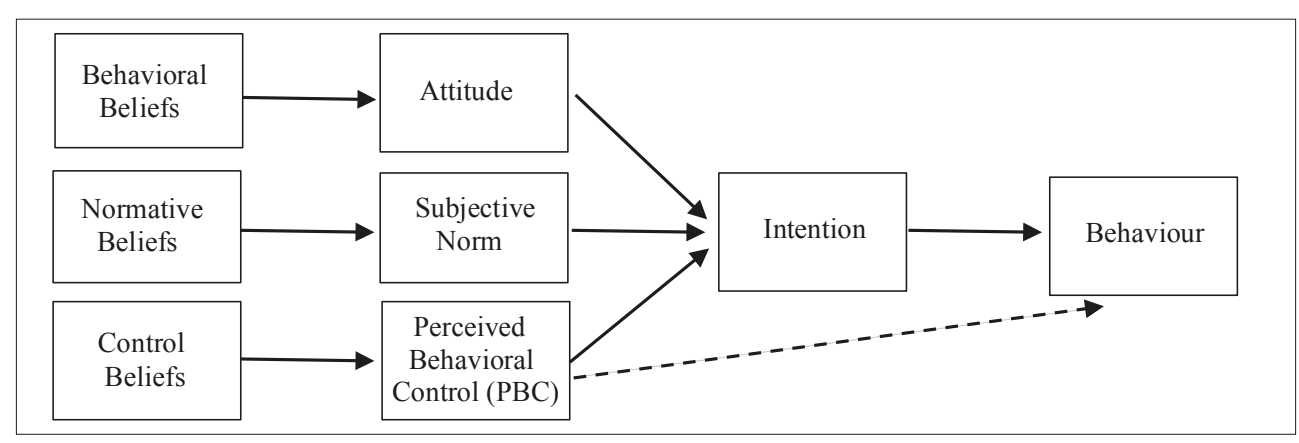

Fuente: Steinmetz, Knappstein, Ajzen, Schimidt y Kabst (2016, p. 220).

Como muestra la figura anterior, las intenciones de comportamiento de un sujeto están condicionadas por sus creencias o convicciones conductuales, es decir, realizando una determinada conducta obtendrá un beneficio o perjuicio para él. Si considera que obtiene un beneficio genera actitudes positivas hacia esa conducta; si considera que le perjudica generará actitudes negativas. Las intenciones de comportamiento también están condicionadas por las creencias o convicciones del sujeto sobre las expectativas que tienen sus referentes sociales sobre si realizará o no la conducta; es lo que se entiende como la presión social que el sujeto percibe sobre él. Por último, las intenciones de comportamiento están condicionadas por las creencias o convicciones del sujeto sobre sus capacidades de controlar la conducta que se concretan en las habilidades con las que se percibe para ello. La teoría y la interacción dinámica de los elementos que la conforman han sido descritas ampliamente en otras publicaciones (Escámez, García, Pérez y Llopis, 2007; Escámez, 2019).

En concreto, la teoría del Comportamiento Planificado de Ajzen la consideramos potente para la construcción de un cuestionario exploratorio de las percepciones de los estudiantes universitarios sobre sostenibilidad porque ofrece las siguientes características: $a$ ) un significado preciso de cada uno de 
los elementos que explican las intenciones de comportamiento (convicciones, actitudes, normas sociales, asumidas por el individuo como normas propias, y habilidades de control de conducta), al mismo tiempo que una determinación de su estructura y de la dinámica de su relación; $b$ ) posibilidad de procedimientos rigurosos de medida de cada uno de los elementos; $c$ ) criterios para poder intervenir técnicamente en la formación y cambio de cada uno de ellos; y d) la determinación de la función y peso de cada uno de los elementos del área para la comprensión de la intención y la predicción de la conducta correspondiente.

Dado que las convicciones conductuales (conocimientos y valores), normativas (presiones sociales) y de control (habilidades para conseguir el resultado previsto) explican los antecedentes de las intenciones, ¿qué tipo de cambio en estos sistemas de convicciones/creencias producen cambios en las actitudes, las normas subjetivas y las habilidades? Investigar el mecanismo a través del cual los sistemas de creencias/convicciones se relacionan con los antecedentes de las intenciones puede ofrecer a los investigadores y a los docentes de la sostenibilidad nuevos conocimientos teóricos y nuevas estrategias y técnicas para la formación y el desarrollo de las intenciones del alumnado como agente de la sostenibilidad.

Así, se podrá alcanzar la propuesta de la Asamblea General de las Naciones Unidas del 25 de septiembre de 2015, conocida como la Agenda 2030 para el Desarrollo Sostenible (ONU, 2015a) que especifica los 17 objetivos cuya finalidad es garantizar una vida sostenible, pacífica, próspera y justa en la tierra para todos, ahora y en el futuro. Por ello, tales Objetivos de Desarrollo Sostenible deben ser considerados como objetivos de aprendizaje para la educación formal en todos sus niveles, desde la infancia hasta la universidad, así como para la educación no formal e informal.

Según la teoría del Comportamiento Planificado, solo la intención predice la conducta bajo las siguientes condiciones: si está referida a la realización de un tipo de acción, con un objetivo preciso, en un contexto determinado y no trascurre un periodo de tiempo largo entre la manifestación de la intención de conducta y la efectiva realización de esta. Cabe destacar, por último, que la intención tiene más potencial de predicción cuando se refiere a un tipo de conductas que a una conducta concreta y cuando se aplica a un conjunto de población que a un individuo. 


\section{Metodología de la investigación}

En las investigaciones sociales, como la que presentamos, es conveniente combinar la metodología cuantitativa con la cualitativa para obtener un conocimiento más profundo del objeto que se investiga. Con este propósito, se utiliza el método empírico-analítico, que nos permite explicar los elementos que inciden en las intenciones de comportamiento; y el hermenéutico-crítico, que nos posibilita comprender los significados de las decisiones y acciones de las personas, a través de la interpretación de sus respuestas.

Respecto al estudio empírico, hemos optado por un diseño de corte descriptivo y exploratorio. Para ello se ha elaborado un cuestionario-escala, atendiendo a las prescripciones de la teoría del Comportamiento Planificado, con las siguientes fases: 1) el punto de partida, las declaraciones manifestadas en las diferentes sesiones por los miembros de los grupos de discusión que nos aportaron un banco amplio de 255 declaraciones; 2) hemos eliminado las declaraciones con el mismo significado o análogo; 3) las declaraciones seleccionadas (104) han sido valoradas por siete expertos/as universitarios en sostenibilidad, con puntuaciones de 1 a 10, y hemos calculado las medias obtenidas para cada una de ellas. Se han eliminado las declaraciones con una media inferior a 8,5 y/o un cociente de variación entre ellas superior al $25 \%$; 4) hemos formulado las declaraciones en items, de acuerdo con las prescripciones de la teoría del Comportamiento Planificado; 5) siguiendo los anteriores criterios de eliminación, nos quedaron 69 ítems que aplicamos como cuestionario-escala piloto a 160 estudiantes de Administración y Dirección de Empresas de otra universidad, ajena a la muestra objeto de investigación. Utilizando el programa Statistical Package for the Social Sciences-23 (Spss23) sometimos las respuestas a análisis estadísticos con la finalidad de llevar a cabo una depuración de los ítems siguiendo los procedimientos para obtener una alta fiabilidad y validez; 6) el análisis de fiabilidad del cuestionario obtiene un alfa de Cronbach de .823; 7) el análisis de validez obtiene siete factores, a cada uno de los cuales se denominó con el nombre de los elementos que conforman la teoría del Comportamiento Planificado: creencias conductuales (conocimientos y valores), creencias normativas, creencias sobre control percibido, actitudes, normas subjetivas, habilidades e intenciones de conducta. Los análisis anteriores nos proporcionaron un cuestionario-escala definitivo 
compuesto por 39 ítems, que aplicamos a la muestra del estudiantado de la Universidad Católica de Valencia San Vicente Mártir y de la Universidad Politécnica de la ciudad de Valencia.

La muestra. La población sometida a la presente investigación es el alumnado del tercer curso del grado de Administración y Dirección de Empresas; entre ambas universidades, según los datos oficiales proporcionados por ambos centros, suman un total de 203 estudiantes. Para conseguir que la muestra sea un subconjunto representativo de la población es preciso concretar el nivel de confianza que queremos que alcancen los datos, optando en esta ocasión por un nivel de confianza del $95 \%$, y un posible error de estimación de un $5 \%$. Para determinar el tamaño de la muestra, utilizamos la fórmula propuesta por Kolmogorov-Smirnov, que queda establecida en 133 estudiantes.

A su vez, dividimos al alumnado de la muestra en función del género: 1) mujeres; 2) varones. Al distribuir la muestra de este modo, se logra que cada género esté representado proporcionalmente a su frecuencia en la población total. La muestra teórica arroja un total de 133 alumnos y alumnas. Sin embargo, como la muestra representativa encuestada se hizo en situación normal de asistentes a las aulas, se administraron 136 cuestionarios, un número ligeramente superior a la distribución teórica, con 69 mujeres y 67 varones.

Para la interpretación de las respuestas dadas por el alumnado en el cuestionario-escala, hemos usado el método hermenéutico. La hermenéutica crítica tiene relevancia metodológica para incorporar en un nuevo orden de racionalidad las motivaciones de las actividades económicas (Conill, 2009). En modo alguno pretendemos justificar ni revivir una hermenéutica pura que desemboque en una visión ontológica del mundo ideal, ajeno a los datos que se obtienen en los protocolos de diagnóstico de las intenciones de comportamiento de los estudiantes como agentes de sostenibilidad. Nuestra postura está a favor de una hermenéutica crítica impura (Conill, 2006) que tiene relevancia metodológica para comprender el sentido de las respuestas del estudiantado. Es el método que consideramos óptimo para interpretar el significado y sentido de las respuestas del alumnado. 


\section{INTENCIONES DE COMPORTAMIENTO DEL ESTUDIANTADO DE LA} Universidad CATÓlica de Valencia y de la Universidad Politécnica de Valencia (España) como agentes de Sostenibilidad

Aunque el cuestionario-escala hace un estudio exploratorio descriptivo de los 7 factores que incluye la teoría del Comportamiento Planificado, en este artículo solo se expone el análisis de los datos y la discusión de los resultados de una de las subescalas: las intenciones del comportamiento (cuadro 1), que incluye cuatro ítems: 2, 5, 13 y 33. La subescala diagnostica las manifestaciones del sujeto sobre sus decisiones de realizar o no determinados comportamientos.

\section{CUADRO 1}

Relación de items que componen la subescala de intenciones de comportamiento

\begin{tabular}{|l|r|r|r|}
\hline \multicolumn{1}{|c|}{ Intenciones de comportamiento } & Adecuadas & Inadecuadas & Indiferentes \\
\hline $\begin{array}{l}\text { 2. Participaré en las redes de comunicación y en los } \\
\text { movimientos sociales para la defensa de los derechos } \\
\text { humanos. }\end{array}$ & $56,1 \%$ & $12,1 \%$ & $31,8 \%$ \\
\hline $\begin{array}{l}\text { 5. La pobreza y la exclusión son asuntos que comba- } \\
\text { tiré activamente. }\end{array}$ & $65,8 \%$ & $7,4 \%$ & $26,8 \%$ \\
\hline $\begin{array}{l}\text { 13. Participaré en los movimientos ciudadanos defen- } \\
\text { sores del medio ambiente. }\end{array}$ & $45,6 \%$ & $20,3 \%$ & $34,1 \%$ \\
\hline $\begin{array}{l}\text { 33. Defenderé el bien común más que el beneficio } \\
\text { propio. }\end{array}$ & $40,4 \%$ & $24,2 \%$ & $35,4 \%$ \\
\hline
\end{tabular}

Fuente: Elaboración propia.

Aplicando el programa estadístico Spss-23 para analizar las respuestas a los ítems anteriores, se obtuvieron los datos siguientes: 
CUADRO 2

Análisis descriptivo de los items de la subescala de intención del comportamiento

\begin{tabular}{|l|l|r|r|r|r|}
\hline \multicolumn{2}{|c|}{} & \multicolumn{1}{|c|}{ Ítem 5 } & \multicolumn{1}{c|}{ Ítem 2 } & \multicolumn{1}{c|}{ Ítem 13 } & \multicolumn{1}{c|}{ Item 33 } \\
\hline \multirow{2}{*}{$\mathrm{N}$} & Válido & 123 & 132 & 123 & 131 \\
\cline { 2 - 6 } & Perdidos & 13 & 4 & 13 & 5 \\
\hline \multirow{2}{*}{ Media } & 3,732 & 3,545 & 3,228 & 3,183 \\
\hline Moda & 4,0 & 4,0 & 4,0 & 4,0 \\
\hline Desviación estándar &, 8305 &, 9440 & 1,0148 &, 9098 \\
\hline Varianza &, 690 &, 891 & 1,030 &, 828 \\
\hline
\end{tabular}

Fuente: Elaboración propia.

\subsection{Análisis de los datos}

Para una mayor claridad en el análisis de los datos y la posterior discusión de los resultados, nos hemos tomado la licencia de agrupar las respuestas, de los cinco grados de la escala de Likert, en los ítems de tres categorías: los que están en desacuerdo (grados 1 y 2), los indiferentes (grado 3 ) y los que están de acuerdo (grados 4 y 5). Los tres ítems que tienen más peso en la subescala intención de comportamiento manifiestan diferencias entre sí. El ítem 5 muestra el compromiso del 65,8 \% del estudiantado con dos de los problemas centrales de la sostenibilidad social: "La pobreza y la exclusión son asuntos que combatiré activamente". Frente a este dato, encontramos al 7,4 \% del estudiantado que no acepta este compromiso. Es preocupante, sin embargo, que el 26,8 \% del alumnado sea indiferente a esos problemas. Tales resultados son mejores que el grado de compromiso que se tiene en la participación ciudadana para la defensa de los Derechos Humanos, como se manifiesta en el ítem 2: "Participaré en las redes de comunicación y en los movimientos sociales para la defensa de los Derechos Humanos", donde el 56,1 \% del estudiantado está de acuerdo y un preocupante $12,1 \%$ se encuentra en desacuerdo, situándose en una posición de indiferencia el 31,8\%. La defensa activa del medio ambiente, que se mide en el ítem 13: "Participaré en los movimientos 
ciudadanos defensores del medio ambiente", genera en el estudiantado un menor compromiso, pues solo está de acuerdo el 45,6\% del alumnado frente al 20,3\% que está en desacuerdo, manifestándose un 34,1\% de manera indiferente. De ahí que no sea extraña la percepción central para la gestión de la sostenibilidad que el alumnado sostiene en el ítem 33: "Defenderé el bien común más que el beneficio propio", con el que está de acuerdo el 40,4\%, en desacuerdo el 24,2 \% e indiferente el 35,4\%, dando estos dos últimos apartados un llamativo 59,6\%. La representación gráfica de estos datos se presenta a continuación (figura 2 ):

FIGURA 2

Representación gráfica de los datos de la sub-escala de intenciones de comportamiento

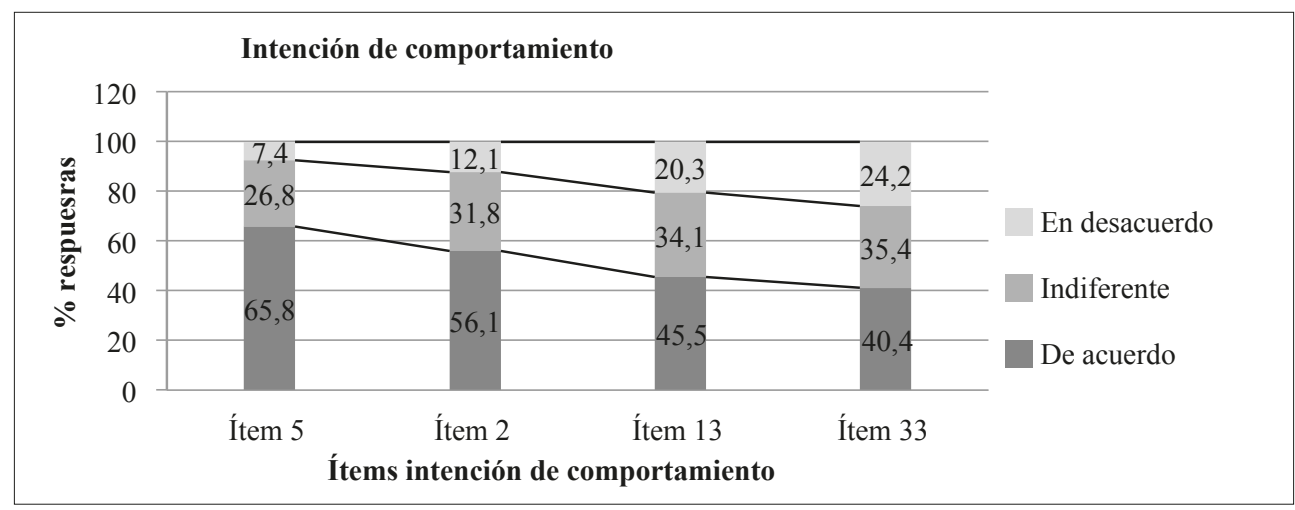

Fuente: Elaboración propia.

\subsection{Discusión de los resultados}

Respecto a las intenciones de comportamiento es mayor el compromiso del estudiantado con la solución de problemas concretos de la sostenibilidad social como la pobreza y la exclusión que con la defensa de los Derechos Humanos, debido quizás al desconocimiento del significado entre el estudiantado, del concepto de Derechos Humanos. Pero en uno y en otro caso predomina la intención de comportamiento positivo sobre el negativo. Valoración positiva que también sucede, en grado mucho menor, en el caso del activismo 
cívico en la defensa del medio ambiente. La intención de comportamiento positivo desciende progresivamente en los ítems con más peso en el factor, el $65,8 \%$, el 56,1 \% y el 40,4\% del estudiantado, respectivamente.

Evaluar bien las intenciones del comportamiento de los estudiantes no es un asunto menor para predecir su conducta futura como agentes de sostenibilidad. Como argumentan Steinmetz, Ajzen y colaboradores (2016), en las últimas décadas, la teoría del Comportamiento Planificado (Ajzen, 1991 y 2012) se ha convertido en un marco para explicar y predecir el comportamiento (Fishbein y Ajzen, 2010). La teoría establece que el principal impulsor del comportamiento es la intención, entendida como una función de las variables motivacionales subyacentes (convicciones, actitudes, normas subjetivas y control percibido).

En cuanto a la resistencia del estudiantado a su implicación en los movimientos sociales, que muestra el ítem 13, pues como se puede observar solo el 45,5\% está dispuesto a participar, a nuestro juicio se debe en gran parte a las estrategias de aprendizaje-enseñanza que son usuales en las universidades españolas, centradas mayoritariamente en las clases magistrales y en las prácticas. Como insiste la Unesco, en su informe Educación para los Objetivos de Desarrollo Sostenible. Objetivos de Aprendizaje (2017), es necesario formar al profesorado para la práctica de una pedagogía transformadora orientada a la acción, que involucre al alumnado en procesos de pensamiento y acción participativos, sistémicos, creativos e innovadores en el contexto de sus comunidades locales y de sus vidas cotidianas, con el objetivo de que se conviertan en agentes de cambio (Fullan, 2002) en un proceso de aprendizaje organizativo que impulse hacia el desarrollo sostenible.

En relación con investigaciones importantes, la ausencia de activismo cívico para la gestión de la sostenibilidad concuerda con la macroinvestigación bianual, auspiciada por la ONU y liderada por Haski-Levental, en los años 2012, 2013 y 2015, en la que se detecta una disminución del activismo del estudiantado universitario en los últimos años en tres de los ítems referidos: a medir las horas dedicadas a actividades de voluntariado, a las donaciones económicas y a la participación en los movimientos sociales. En la última encuesta (Haski-Levental y Concato, 2016), se detecta, además, una diferencia notable en relación con las encuestas anteriores dada la inclusión de estudiantes de pregrado, como es la población que estudia nuestra investigación. 
En relación con el párrafo anterior, invita a la reflexión y merece nuestro comentario el alto porcentaje de estudiantes que en la escala de Likert se autositúan, en los tres ítems, en la posición de indiferentes: en el ítem 5, el 26,8 \%; en el ítem 2, el 31,8\%; y en el ítem 13, el 34,1\%. Esto nos hace pensar que algo está fallando en la formación universitaria sobre los aspectos relacionados con la sostenibilidad, como declara un miembro de los grupos de discusión: “...Yo, concretamente creo que... hablando de la universidad, no se le da mucha importancia a la sostenibilidad y, al final del día, lo importante es que tienes que ser eficiente y ya está" (M3).

Nos aventuramos a conjeturar que esa indiferencia del alumnado se debe a dos circunstancias principales: la primera es que se está produciendo en nuestro tiempo un cambio en la mentalidad del alumnado a favor del compromiso con la sostenibilidad, pero que tal cambio le genera perplejidad y desconcierto; la segunda circunstancia es el divorcio entre lo que se sabe y lo que se hace: un problema clásico planteado de modo preciso por Kohlberg y Candee (1984), pero que viene desde los griegos, cuando se planteaban si se puede enseñar/aprender la virtud. Como podemos inferir, se trata de un problema que transita toda la historia de la educación moral, llegando incluso hasta nuestros días. En todo caso, la autopercepción de indiferencia ante un problema tan importante como la intención de gestionar la sostenibilidad es una cuestión central para la formación del estudiantado, pues manifiesta o bien la endeblez de las convicciones o bien la insuficiencia de los conocimientos para llevar a cabo los comportamientos, como defiende la teoría de la Formación del Carácter cuando postula que para formar el carácter lo más adecuado es aplicarse a la realización de acciones (Escámez, 2003).

En cuanto a las intenciones que mantienen los estudiantes en el ítem 33, "Defenderé el bien común más que el beneficio propio", es alentador que sea mayor el número de estudiantes que están de acuerdo que los que están en desacuerdo, aunque no debemos perder de vistas que entre estos últimos y los indiferentes alcanzan un rotundo 59,6 \%. Desde el punto de vista del bien común, la sostenibilidad no es un bien limitado a un individuo o a unos cuantos individuos o a unos Estados nación concretos. La concepción de la sostenibilidad como bien común universal reafirma su dimensión colectiva como tarea social común, como responsabilidad compartida y compromiso con la solidaridad (Unesco, 2015). 
Consideramos que es necesario dar a conocer los grandes documentos de nuestro tiempo al alumnado universitario, como se puso en evidencia cuando en los grupos de discusión se afirma que no se hace referencia alguna al conocimiento del Pacto Mundial de la ONU (2004), a los Principios para una Educación Responsable en Gestión (2007), al informe de Bokova Replantear la educación. Hacia un bien común universal (2015) y a los Objetivos del Desarrollo Sostenible. Objetivos de Aprendizaje (UNESCO, 2017), entre otros muchos documentos.

4. ¿INFLUYE EL GÉNERO EN LAS INTENCIONES DE COMPORTAMIENTO?

Uno de los aspectos que nos ha interesado analizar en cuanto a las intenciones de comportamiento de los estudiantes en la gestión de la sostenibilidad es la existencia o no de diferencias en función del género. La distribución de la muestra según género queda de la siguiente manera:

CUADRO 3

Distribución de la muestra en función del género

\begin{tabular}{|l|l|r|r|r|r|}
\hline \multicolumn{2}{|c|}{} & \multicolumn{1}{|c|}{ Frecuencia } & Porcentaje & Porcentaje válido & Porcentaje acumulado \\
\hline \multirow{4}{*}{ Válido } & Hombres & 67 & 49,26 & 49,26 & 49,26 \\
\cline { 2 - 6 } & Mujeres & 69 & 50,74 & 50,74 & 50,74 \\
\cline { 2 - 6 } & Total & 136 & 100,0 & 100,0 & \\
\hline
\end{tabular}

Fuente: Secretarías de las facultades de Administración y Dirección de Empresas.

A continuación presentamos cada uno de los ítems que componen la subescala de intención de comportamiento para determinar si hay diferencias notables según el género. Hemos atendido a dos criterios estadísticos importantes: la diferencia de puntuación media entre mujeres y varones y la diferencia en la moda dentro de una escala Likert de cinco grados (en qué grado se concentran las respuestas del alumnado). Dado que, en el preanálisis que hemos realizado de la muestra del alumnado de las dos universidades de Valencia, es frecuente que el valor mayor o menor de la moda se deba a la variación de un 
número mínimo de estudiantes, hemos decidido tener en cuenta, sobre todo, la media de puntuaciones de varones y mujeres, sombreando el ítem correspondiente, tal como aparece a continuación.

CUADRO 4

Diferencias en intención del comportamiento en función del género

\begin{tabular}{|c|c|c|c|c|c|c|}
\hline \multicolumn{3}{|c|}{ Género } & Ítem 5 & Ítem 2 & Ítem 13 & Ítem 33 \\
\hline \multirow{3}{*}{ Hombre } & \multirow{2}{*}{$\mathrm{N}$} & Válidos & 59 & 64 & 58 & 63 \\
\hline & & Perdidos & 8 & 3 & 9 & 4 \\
\hline & \multicolumn{2}{|c|}{$\begin{array}{l}\text { Media } \\
\text { Moda }\end{array}$} & $\begin{array}{r}3.58 \\
4 \\
\end{array}$ & $\begin{array}{r}3.22 \\
3 \\
\end{array}$ & $\begin{array}{r}2.97 \\
3 \\
\end{array}$ & $\begin{array}{r}2.95 \\
3 \\
\end{array}$ \\
\hline \multirow{4}{*}{ Mujer } & \multirow{2}{*}{$\mathrm{N}$} & Válidos & 64 & 68 & 65 & 68 \\
\hline & & Perdidos & 5 & 1 & 4 & 1 \\
\hline & \multirow{2}{*}{\multicolumn{2}{|c|}{$\begin{array}{l}\text { Media } \\
\text { Moda }\end{array}$}} & 3.88 & 3.85 & 3.46 & 3.40 \\
\hline & & & 4 & 4 & 4 & 4 \\
\hline
\end{tabular}

Fuente: Elaboración propia.

\subsection{Análisis de los datos}

Como puede observarse en el cuadro anterior, todos los ítems presentan diferencias de media en el compromiso (intención de comportamiento) a favor de las mujeres con respecto a los varones. En el ítem 5, "La pobreza y la exclusión serán asuntos que combatiré activamente", coincide la moda de mujeres y varones siendo la variación de la media no significativa. En los ítems 2, "Participaré en las redes de comunicación y en los movimientos sociales para la defensa de los Derechos Humanos"; ítem 13, "Participaré en los movimientos ciudadanos defensores del medio ambiente"; y el ítem 33, "Defenderé el bien común más que el beneficio propio", encontramos diferencia en la moda de las mujeres, con valor 4 (de acuerdo), frente a la moda de los varones con valor 3 (indiferente). Así mismo, las diferencias de medias son significativas en los 3 ítems, puesto que las mujeres presentan en el ítem 2 una media de 3,85 frente a 3,22 de los varones; en el ítem 13, la media de las 
mujeres es de 3,46 frente a 2,97 de los varones; y en el ítem 33, la media de las mujeres es de 3,40 frente a 2,95 de los varones.

\subsection{Discusión de los resultados}

Los resultados de nuestra investigación son coherentes con los de la macroencuesta mundial de Haski-Leventhal, Pournader y McKinnon (2015) que prueba que las mujeres tienen un mayor compromiso ético para implicarse en la ayuda a la comunidad y en las necesidades de la gente, así como en la mejora del medio ambiente. Igualmente son consistentes en vivir de acuerdo con sus valores y están dispuestas a cambiar sus hábitos de comportamiento si es necesario. En cuanto a sus actitudes, evalúan positivamente las conductas de las empresas y de las personas que trabajan para la mejora de la sociedad y del medio ambiente, así como la posibilidad de construir un mundo mejor en el que sea posible el gozo de los derechos sociales por todos los ciudadanos. En análogo sentido se manifiestan las revisiones realizadas por Haus, Steinmetz, Isidor y Kabst (2013).

Los datos de nuestra investigación nos retrotraen al debate generado en la década de 1980. Gilligan (1982), discípula de Kohlberg, introdujo un debate académico sobre si el enfoque moral está determinado por el género: los hombres y las mujeres tienen diferentes orientaciones de vida y diversos puntos de vista sobre cuestiones y dilemas éticos fundamentales, que defendía Gilligan, o si el desarrollo moral se debe a la edad, al desarrollo del juicio y a la perspectiva social que adopta el sujeto como defendía Kohlberg (1981, 1984). Gilligan difiere de algunas de las interpretaciones que Kohlberg hizo de los resultados de su investigación sobre el desarrollo moral. Es aquí donde nace la propuesta de la denominada Ética de la Responsabilidad y el Cuidado, que tanta importancia tiene para la gestión de sostenibilidad (Paya y Rubio, 2018). La viveza y perdurabilidad del debate se puede observar en la reciente publicación, Una mirada femenina de la educación moral (2018), coordinada por las profesoras Buxarrais y Vilafranca, en la que se muestra que las mujeres son más sensibles que los varones a las necesidades de los demás y del medio ambiente. Nuestra interpretación de los datos concuerda con la ética y 
pedagogía para el cuidado, así como con el enfoque del desarrollo de capacidades humanas.

Respecto a la ética y educación para el cuidado, Noddings (2006), la pensadora más importante de la ética para el cuidado y pionera de su aplicación al ámbito educativo, ha desarrollado una amplia bibliografía con la premisa de crear un currículo ajeno a las dicotomías sexistas y que afronte, entre otras, la necesidad de dar respuesta a las necesidades humanas relativas a la búsqueda de un sentido existencial, la formación para la atención a las personas vulnerables, la protección del medio ambiente y la convivencia pacífica (2002).

Respecto al enfoque que considera el desarrollo como crecimiento de capacidades humanas frente a su consideración, predominante hoy en día, como crecimiento del producto interior bruto, los autores más significativos de este enfoque, A. Sen (premio Nobel de Economía) y M. C. Nussbaum (premio Príncipe de Asturias en Ciencias Sociales), argumentan que el vigor de las sociedades democráticas y de las libertades civiles, como espacios de libertad (Sen, 2000), así como la calidad del entorno natural y la salud de los ecosistemas, se justifican mejor atendiendo a los valores de la dignidad, respeto e igualdad de mujeres y varones: "Ante las cuestiones de la calidad y la sostenibilidad medioambientales, el enfoque de las capacidades tiene una serie de ventajas bien definidas sobre otras perspectivas que cuentan con especial predicamento en la economía medioambiental actual" (Nussbaum, 2012, p. 193).

\section{Conclusiones}

Lo que predice el futuro comportamiento del estudiantado para la gestión de la sostenibilidad es la intención de conducta, de acuerdo con la teoría del Comportamiento Planificado que hemos utilizado como marco teórico para construir el instrumento de diagnóstico. En las respuestas a los 4 ítems, el estudiantado muestra un compromiso (intención) positivo para la acción mayor que el estudiantado que rechaza tal compromiso. La intención de comportamiento es mayor cuando está referido a la solución de problemas sociales concretos de la sostenibilidad que a otros problemas con una formulación más abstracta, como la defensa de los Derechos Humanos, y desciende fuertemente cuando tiene que ser concretado con la participación en movimientos 
sociales para hacerla efectiva. La intención de comportamiento de las mujeres para la gestión de la sostenibilidad es mayor que la de los varones. Un problema fundamental que se detecta es el número de alumnos que se autositúan en una posición de indiferencia; asunto que urge la necesidad de plantear nuevas estrategias de aprendizaje-enseñanza universitarias abocadas a la acción para la trasformación social y la búsqueda del bien común. Las universidades estudiadas tienen que reformular su estrategia general de formación del alumnado, los planes de estudio de la titulación y la formación del profesorado, tanto en conocimientos de sostenibilidad como en técnicas pedagógicas de aprendizaje-enseñanza para promover en los estudiantes la intención de su gestión efectiva.

Somos conscientes de las limitaciones de la presente investigación: analizamos solo cuatro ítems para diagnosticar las intenciones del comportamiento del estudiantado y la muestra está circunscrita al tercer curso académico del grado de Administración y Dirección de Empresas de dos universidades. Tampoco hemos ofrecido programas educativos para la mejora de aquellos estudiantes que tienen un compromiso (intenciones) negativo e indiferente. Esperamos subsanar esas deficiencias en el futuro ampliando la muestra a todo el alumnado de Administración y Dirección de Empresas en estas universidades y en otras universidades españolas y, si es posible, en proyectos de investigación compartidos con universidades de otros países, así como proponiendo programas educativos concretos para la mejora de las intenciones de comportamiento del estudiantado como agente de sostenibilidad. Sin embargo, creemos que la fase de un diagnóstico exploratorio es necesaria y que nos hayamos centrado en las intenciones de comportamiento es importante porque así podemos predecir las conductas posteriores del alumnado en su futuro desarrollo personal y profesional en relación con un tema básico y prioritario de la educación actual.

\section{BibliografíA}

AJzen, I. (1991). The theory of planned behavior. Organizational Behavior and Human Decision Processes 50, 179-211. 
AuzEn, I. (2012). The theory of planned behavior, en P. Lange, A. Kruglanski y E. T. Higgins (eds.), Handbook of theories of social psychology (pp. 438459). Londres: Sage.

Bокоva, I. (2015). Replantear la educación. ¿Hacia un bien común mundial? París: Unesco.

BuXARrais, M. R. y Vilafranca, I. (2018) (coords.). Una mirada femenina sobre la educación moral. Bilbao: Desclée.

Conill, J. (2006). Ética hermenéutica. Madrid: Tecnos.

Conill, J. (2009). Por una economía hermenéutica de la pobreza, en A. Cortina y G. Pereira (eds.), Pobreza y Libertad. Erradicar la pobreza desde el enfoque de Amartya Sen (pp. 151-162). Madrid: Tecnos.

EscÁmez, J. (2003). Pensar y hacer hoy educación moral. Teoría de la Educación. Revista interuniversitaria 15, 21-31.

Escámez, J., García, R., Pérez, C. y Llopis, A. (2007). El aprendizaje de valores y actitudes. Teoría y práctica. Barcelona: Octaedro-OEI.

EsCÁMEZ, J. (2019). La perspectiva cognitiva para la comprensión de las intenciones y la predicción de las conductas del estudiantado como agente de sostenibilidad, en A. D. Arrufat y R. Sanz, La ciudadanía europea como labor permanente (pp. 221-232). Valencia: Tirant lo Blanch.

Fishbein, M. y Ajzen, I. (2010). Predicting and changing behavior: The reasoned action approach. Nueva York: Taylor \& Francis.

Fullan, M. (2002). Las fuerzas del cambio. Explorando las profundidades de la reforma educativa. Madrid: Akal.

Gilligan, C. (1982). In a different voice: Psychogical theory and women's development. Cambridge: Harvard UniversityPress.

Haski-LeVenthal, D. (2012). Corporate responsibility and responsable management education in the eyes of MBA Students. Nueva York: United Nations PRME.

HASKi-LeVENTHAL, D. (2013). MBA students around the world and their attitudes towards responsable management. Sydney: Macquarie University. Haski-Leventhal, D. (2014). MBA student values, attitudes and behaviors: a cross-cultural comparison of PRME signatory schools. SAM. Advanced Management Journal 79(4), 29-41. 
Haski-Leventhal, D., Pournader, M. y McKinnon, A. (2015). The Role of Gender and Age in Business Students' Values, CSR Attitudes, and Responsible Management Education: Learnings from the PRME International Survey. Journal of Business Ethics 146(1), 219-239.

Haski-Leyenthal, D. y Concato, J. (2016). The State of CSR and RME in Business Schools and the Attitudes of their Students. Sydney: Macquarie University.

Haus, I., Steinmetz, H., Isidor, R. y Kabst, R. (2013). Gender effects on entrepreneurial intention: A meta-analytical structural equation model. International Journal of Gender \& Entrepreneurship 5, 130-156.

Herdenson, J. A., Bieler, A. y Mckenzie, M. (2017). Climate change and the Canadian higher education system: An institutional policy analysis. Canadian Journal of Higher Education 47(1), 1-26.

Kohlberg, L. (1981). Essays on Moral Development. Vol. 1: The Philosophy of Moral Development. San Francisco: Harper and Row.

Kohlberg, L. \& CAndeE, D. (1984). The relatioship of moral judgment to moral action. En L. Kohlberg. Essays on Moral Development. Vol. 2: The Psychology of Moral Development (pp. 499-581). San Francisco: Harper and Row.

Lozano, J. F. (2012). Educating Responsible Managers. The Role of University Ethos. Journal of Academic Ethics 10(3), 213-226.

Noddings, N. (2002). Educating moral people. A caring alternative to character education. Nueva York: Teachers College Press.

Noddings, N. (2006). Critical lessons. What our schools should teach. Nueva York: Cambridge University Press.

Nussbaum, M. C. y Sen, A. (1996) (compiladores). La calidad de vida. México: Fondo de Cultura Económica.

Nussbaum, M. C. (2012). Crear capacidades. Propuestas para el desarrollo humano. Barcelona: Paidós.

ONU (2004). Pacto Mundial para el Desarrollo de un Mundo Sostenible. Recuperado de: <http://www.pactomundial.org/global-compact/>.

ONU (2007). Principios para una Educación Responsable en Gestión. Recuperado de: $<$ http://www.unprme.org/resource-docs/EDUCACIONRESPONSABLEPRMEencastellano.pdf $>$. 
ONU (2015a). Objetivos de Desarrollo Sostenible. 17 Objetivos para transformar nuestro mundo de la Agenda 2030. Recuperado de: <http://www. un.org/sustainabledevelopment/es/objetivos-de-desarrollo-sostenible/>.

ONU (2015b). Convención Marco sobre el Cambio Climático. Recuperado de: <http://unfccc.int/resource/docs/2015/cop21/spa/109s.pdf $>$.

PayÁ, M. y Rubio, L. (2018). Carol Gilligan. En una voz diferente, en M. R. Buxarrais e I. Vilafranca, Una mirada femenina de la Educación Moral (pp. 131-147). Bilbao: Desclée.

Sederevičiūtė-PaČiauskiené, Z., Žilinskaitė-Vytiené, V. y Valantinaité, I. (2017) A three-dimensional approach in Education for Sustainable Future. Problems Of Sustainable Development 12(1), 63-69.

SEN, A. (2000). Desarrollo y libertad. Barcelona: Planeta.

Seto-Pamies, D. y Papaoikonomou, E. (2016). A Multi-level Perspective for the Integration of Ethics, Corporate Social Responsibility and Sustainability (ECSRS) in Management Education. Journal of Business Ethics 136, (3), 523-538.

Steinmetz, H., Davidov, E. y Schmidt, P. (2011). Three approaches to estimate latent interaction effects: Intention and perceived behavioral control in the theory of planned behavior. Methodological Innovations Online 6, 95-110.

Steinmetz, H., Knappstein, M., Ajzen, I., Schmidt, P. y Kabst, R. (2016). How Effective are Behavior Change Interventions Based on the Theory of Planned Behavior? A Three-Level Meta-Analysis. Zeitschrift für Psychologie 224(3), 216-233.

Unesco (2017). Educación para los Objetivos de Desarrollo Sostenible. Objetivos de aprendizaje. París: Unesco.

Williams, O. F. (2014). The United Nations Global Compact: What Did It Promise? Journal of Business Ethics 122(2), 241-251. 
